\title{
Biblioteca virtual cooperativa descentralizada con software libre Greenstone
}

\author{
Por Dominique Babini, Florencia Vergara-Rossi, Flavia Medici y Jessica González
}

\begin{abstract}
Resumen: Trabajar con software libre fue una decisión sencilla para crear la Red de Bibliotecas Virtuales de Ciencias Sociales de América Latina y el Caribe hace 10 años, pues el Consejo Latinoamericano de Ciencias Sociales (Clacso) es una red académica de 195 centros de investigación en 21 países de la región que está comprometida con el uso de plataformas libres y con el acceso abierto a los textos digitales de sus investigaciones. En este artículo se describe cómo se desarrolló y cuáles son los procesos de trabajo en esta biblioteca virtual cooperativa que funciona con software libre Greenstone para la sala de lectura, con buscador avanzado, y con software libre Plone para la interfaz gráfica. Ambos permiten la gestión descentralizada de contenidos: 9.000 textos digitales (libros, artículos de revistas, documentos de trabajo, ponencias) y bases de datos.
\end{abstract}

Palabras clave: Bibliotecas virtuales, Bibliotecas digitales, Software libre, Greenstone, Plone, ISIS, Edición electrónica, Clacso, Ciencias sociales, América Latina.

Title: Use of the open source software Greenstone to develop a decentralized cooperative digital Iibrary

Abstract: When the Latin America and the Caribbean Social Sciences Network of Virtual Libraries was created 10 years ago, it was an easy decision to work with open software because the Latin America and the Caribbean Social Science Council (Clacso), an academic network with 195 member institutes located in 21 countries, promotes the use of open software and open access to communicate and exchange research results in digital formats. The development and daily operations of this cooperative digital library are described, including Greenstone for the reading room with advanced search capacity and Plone for the graphical interface; both allow decentralized content management with 9.000 full text publications (books, journal articles, working documents, papers) and databases.

Keywords: Digital libraries, Virtual libraries, Open software, Greenstone, Plone, ISIS, Electronic publishing, Clacso, Social sciences, Latin America.

Babini, Dominique; Vergara-Rossi, Florencia; Medici, Flavia; González, Jessica. "Biblioteca virtual cooperativa descentralizada con software libre Greenstone". En: El profesional de la información, 2008, enero-febrero, v. 17, n. 1, pp. 64-70.

DOI: 10.3145/epi.2008.ene.07

\section{Introducción}

EL CONSEJO LATINOAMERICANO DE CIENCIAS SOCIALES, Clacso, es una red de centros de investigación y docencia en ciencias sociales ubicados en 21 países de América Latina y el Caribe.

Resultó siempre muy difícil hacer circular publicaciones en for- mato impreso para una red de casi 200 centros tan dispersos geográficamente, con una creciente producción académica. Esto se debe a las limitaciones propias de este formato: bajas tiradas de libros y revistas académicas, elevados costos de correo, complicaciones en préstamos interbibliotecarios entre distintas ciudades y países, y la falta de difusión impresa de documentos de trabajo. Asimismo, con el formato impreso es difícil llegar al público interesado en conocer los aportes académicos para comprender mejor los problemas y necesidades sociales, políticas y culturales de América Latina. Debido a esta dificultad, se aprovechó el surgimiento de la Web para ir organizando la distribución del formato digital de los libros, revistas y documentos de 
trabajo producidos por los centros de investigación y docencia de esta red académica.

Hace 10 años surgió la Red de Bibliotecas Virtuales de Ciencias Sociales de América Latina y el Caribe $^{1}$ de Clacso que funciona con software libre Greenstone ${ }^{2}$. Este programa fue creado por la Universidad de Waikato, en Nueva Zelanda, para formar colecciones de textos digitales con un servicio de buscador avanzado, que realiza la búsqueda en texto completo y metadatos. Para la interfaz gráfica de la biblioteca virtual se ha optado por el software libre Plone $e^{3}$ que permite realizar cambios de formato y contenidos vía web de forma totalmente descentralizada.

\section{"La decisión de trabajar con software libre fue la más apropiada para una red académica regional que contempla distintas realidades económicas y culturales en cada país"}

Hoy sabemos que el desarrollo de una biblioteca virtual cooperativa es el camino más adecuado para acelerar y ampliar la visibilidad e impacto de los resultados de las investigaciones. Por un lado, fue una forma de apoyo al desarrollo de nuevos conocimientos y a las actividades de estudio o docencia $y$, por el otro, una herramienta para la sociedad que utiliza cada vez más la web en busca de respuestas a las preguntas que plantea su quehacer profesional. A su vez, la decisión de trabajar con software libre fue definitivamente la más apropiada para una red académica regional ya que contempla las distintas realidades económicas y culturales de cada país de América Latina y el Caribe.

\section{Trabajando con Greenstone}

A partir de la decisión de difundir virtualmente la producción académica, se comenzó a pensar en la elección de la plataforma más adecuada. Teniendo en cuenta las políticas institucionales, los costos, y la necesidad de descentralizar los procesos a medio plazo, se buscó el software libre que mejor se adaptara a estas condiciones y que organizara de forma eficiente las publicaciones digitales de la red.

Por esta razón se eligió Greenstone para desarrollar íntegramente la sala de lectura de la biblioteca virtual. Esta sala ofrece 9.000 textos completos de acceso libre: artículos de revistas académicas, libros, documentos de trabajo, ponencias en congresos, tesis y el año próximo incluirá también los contenidos de tres bases de datos, originalmente desarrolladas en software libre ISIS: referencias bibliográficas, proyectos de investigación e investigadores de Clacso. Greenstone es un conjunto de programas diseñado para crear y difundir colecciones digitales, de código abierto, por lo que permite modificar tanto su apariencia como sus funciones, adaptándolo a las necesidades concretas.

\section{"Greenstone es un} conjunto de programas en código abierto para crear y difundir colecciones digitales"

Este conjunto de programas funciona tanto en forma local como en servidores web y se pueden realizar CDs con el motor de búsqueda incorporado; se presenta en diferentes versiones: Windows, Linux, Solaris y Mac, y ocupa poco espacio. La información se organiza en colecciones y tanto éstas como el resultado de las búsquedas se definen con una estructura propia. Además indexa archivos de diferentes formatos: texto, vídeos, bases de datos, música, fotos, páginas web y e-mails. A su vez, trabaja con metadatos, que pueden ser incorporados manual o automáticamente y son éstos los que le permiten elaborar listados para navegar por la información en cada colección, con la ventaja de que procesa toda la información en $\mathrm{xml}^{4}$. Greenstone tiene incorporado el sistema OAI (Open Archives Initiative) para transferencia de datos desde y hacia colecciones remotas para formar grandes redes de información de textos digitales.

Contiene una interfaz del bibliotecario, diseñada para definir y procesar colecciones en forma local en un ordenador. En nuestro caso utilizamos directamente líneas de comando por consola en un servidor Linux, por lo que se llevan a cabo dos procesos, generalmente realizados por distintas personas, ya que primero se trata el texto y luego se agrega a una colección ya en línea.

\section{Estructura de las colecciones}

La Red de Bibliotecas Virtuales de Ciencias Sociales de América Latina y el Caribe está pensada con un doble objetivo: por un lado es un servicio a los usuarios de la web que buscan textos de ciencias sociales y a la vez es un servicio a los centros miembros de Clacso que buscan una mayor visibilidad y citaciones de sus publicaciones digitales. En Greenstone se crean colecciones para cada institución y para cada tipo de documento.

Desde la página principal de la biblioteca virtual el usuario tiene tres posibilidades para realizar su búsqueda: en toda la sala de lectura, por tipo de documento o por la colección de un centro de investigación en particular. Cada colección posee su propia página principal donde se encuentra la información general, el logo institucional y los datos parti- 


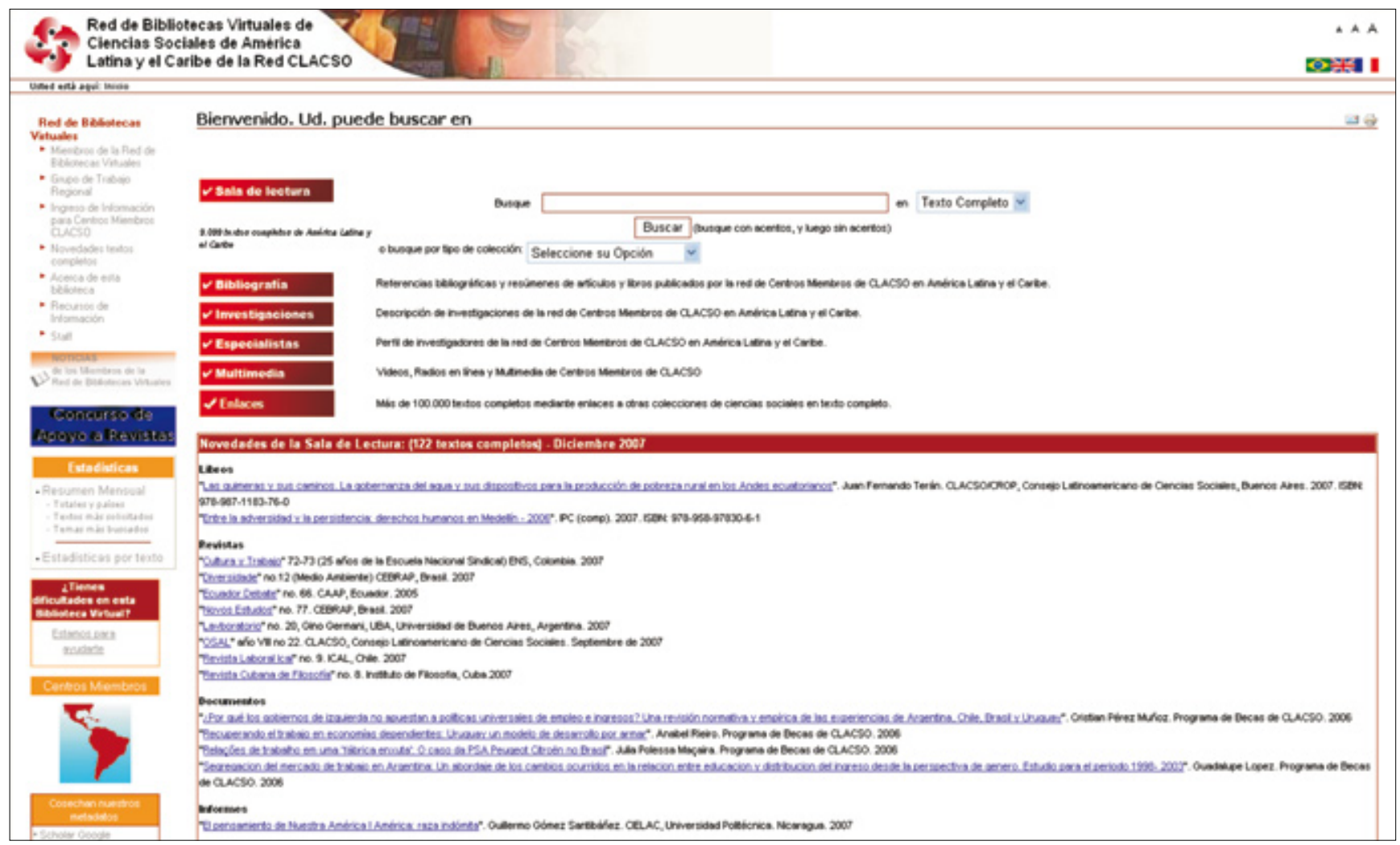

Web de la Red de Bibliotecas Virtuales de Ciencias Sociales de América Latina y el Caribe de Clacso

culares para contactar. Además, se ofrece una explicación de las formas de buscar y las condiciones de uso establecidas por la licencia Creative Commons $^{5}$ que protege los contenidos de la biblioteca virtual.

La búsqueda se puede hacer de dos formas: tema);

- por metadatos (autor, título y

- por un concepto en el texto completo de todas las publicaciones y dentro de cada colección, en listados por autor y por título.

Desde ambas se puede acceder al texto completo.

En esta biblioteca la información se procesa en html, lo que permite cortar el texto en capítulos y realizar así una catalogación analítica de los libros y revistas. A cada parte se le consignan sus propios metadatos, que se utilizan a su vez para crear automáticamente la cita bibliográfica.

La razón principal por la cual procesamos cada artículo de revista y cada capítulo de libro es que al realizar una consulta se recuperan los distintos capítulos por separado y se evita así que los usuarios se vean obligados a bajar el extenso archivo de un libro o una revista completa. De esta forma se simplifica el acceso a la información ya que la mayoría de los usuarios de América Latina y el Caribe no cuentan con banda ancha y por lo general están interesados sólo en uno de los artículos o capítulos del texto. Además con html es sencillo insertar la portada, las imágenes y cuadros que el texto posea. La finalidad de trabajar así e incluir en el encabezado de cada capítulo el enlace al texto en su formato original (pdf, rtf, doc, etc.), es poder ofrecer a los usuarios la versión en pantalla y la posibilidad de que puedan guardarse una copia para imprimir.

Las colecciones funcionan de forma independiente pero pueden enlazarse unas con otras utilizando la opción que en Greenstone se llama super collection. De esta forma se puede hacer una búsqueda en todas las colecciones a la vez o en aquellas que se programen. Por tipo de documento realizamos diferentes súper búsquedas:

- En todas las colecciones.

- Artículos de revistas académicas.

- Libros y documentos de trabajo.

- Ponencias de congresos.

- Tesis.

Se facilita así la consulta en tiempo y forma, porque el usuario puede realizar una sola búsqueda sobre las 190 colecciones con las que contamos en este momento, en lugar de recorrerlas una por una para encontrar la información que necesita.

\section{Transferencia de tecnología}

La gran ventaja de trabajar con colecciones es que la información puede estar alojada en un servidor y ser ofrecida en forma de enlace desde cualquier página. Esto es im- 
portante para la red de bibliotecas virtuales de Clacso porque no todas las instituciones que la conforman cuentan con servidores propios para instalar esta plataforma, ni con el personal capacitado para desarrollarla. Entonces, las colecciones de los centros cooperantes, que estén alojadas en los servidores de la Oficina coordinadora de la biblioteca virtual cooperativa, pueden ser ofrecidas por los centros como enlaces desde sus webs institucionales. De esta forma el centro puede dar acceso a toda su producción académica ordenada dentro de este poderoso buscador como un servicio más.

Sin embargo, algunos centros ya han comenzado a instalar su propia biblioteca digital utilizando Greenstone. Para poner en práctica la estructura de cada colección se les proporciona el archivo de configuración, que se puede copiar exacto o se le pueden hacer los cambios que se consideren necesarios. Para modificarlo se trabaja sobre el archivo de configuración de cada colección con cualquier editor de texto plano. En este sentido también se realizan capacitaciones con manuales en castellano, se brinda apoyo vía correo electrónico y chat con los centros miembros.

\section{OAI}

Los centros miembros que tienen otras bases de datos en texto completo pueden incorporarse a la red de bibliotecas virtuales por medio de $O A I$, un protocolo de intercambio que permite compartir la información independientemente de la plataforma que cada centro tenga. Se realizaron pruebas con la University of Texas - Latin American Network Center (UT-Lanic) y en este momento estamos experimentando con la Universidad Autónoma del Estado de México, que tiene un software propio. Este proceso se realiza únicamente a nivel de metadatos, e incluimos dentro de ellos la dirección web del documento original. De esta manera los portales se enriquecen, ofreciendo a sus usuarios una mayor variedad y cantidad de información proveniente de toda la red.

Cuando se utilizan programas libres se deben considerar los costos de adaptación a las necesidades locales y de capacitar a quienes deben utilizarlo para actualizar las colecciones. La ventaja de que todos los miembros de la red utilicemos la misma plataforma es que de esta forma se pueden realizar transferencias e intercambios de los conocimientos adquiridos por cada uno.

\section{PI05}

Desde la secretaría ejecutiva de Clacso se está impulsando un proceso de descentralización del trabajo hacia los centros de investigación de ciencias sociales que conforman la red. Con este objetivo, en la $O f$ cina coordinadora de la biblioteca virtual cooperativa comenzamos a desarrollar distintas herramientas para facilitar la incorporación de la producción de cada uno de los centros miembros a la sala de lectura de la biblioteca.

En este marco se desarrolló una interfaz gráfica de usuario llamada PI05. Fue específicamente pensada como un sistema para facilitar la preparación de los documentos y el trabajo necesario para generar los tres archivos requeridos por Greenstone cuando se procesan los textos en formato html: html, xml y jpg. Éstos son necesarios para la inclusión de documentos de trabajo, publicaciones periódicas o libros de cada uno de los centros miembros de Clacso en sus respectivas colecciones, para ser luego presentados en la sala de lectura.

Aunque la interfaz se encuentra aún en etapa beta funcional (sujeta a mejoras futuras), se logró agilizar la técnica de trabajo, ya que hizo más simple tanto la metodología para ingresar metadatos en cada parte del documento, como la tarea de darle forma con etiquetas html. Esto significó un importante avance en la descentralización del trabajo de incorporación de libros, documentos y artículos.

PI05 se puede bajar libremente, junto con su manual de uso, de la sección "Recursos de información" de la biblioteca. Esta interfaz ofrece diferentes esquemas de metadatos predefinidos para cada tipo de publicación que pueden ser adaptados a distintas necesidades, tanto para publicaciones específicas como para utilizarlos en otros servicios. Así se simplifica enormemente la inclusión de metadatos.

“PI05 permitió que cada vez sean más los centros cooperantes que trabajan a distancia preparando sus propios documentos para Greenstone"

Esta interfaz gráfica presenta dos grandes partes. En la primera se completan los datos generales de la publicación, tanto sus metadatos como la presentación general del documento; y en la segunda se divide el texto en secciones y se trabaja de forma analítica cada capítulo o contribución del documento general. Esta segunda parte está pensada para los libros colaborativos, que tienen capítulos de distintos autores, así como para las publicaciones periódicas académicas, que generalmente tienen división de artículos por autores o secciones especiales que engloban distintos documentos.

La finalidad de este proceso de división en secciones es que se genere automáticamente en Greenstone un índice interactivo del texto con sus distintos capítulos. De esta 


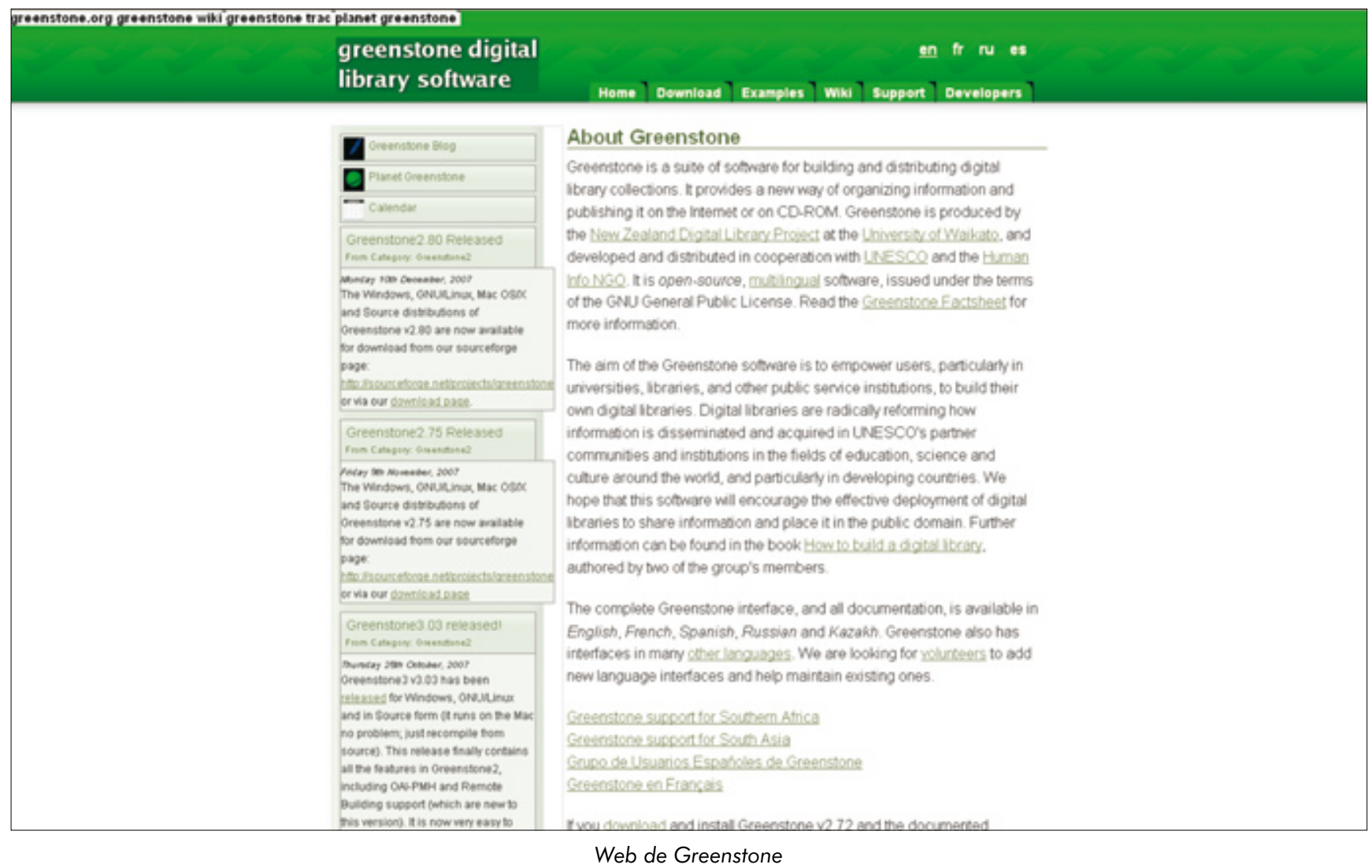

manera, al realizar una búsqueda el resultado serán capítulos específicos sobre un tema, un autor o un título, y no todo el volumen completo. Se trabaja en dos planos, por un lado se incluyen los metadatos específicos de la sección y por el otro, se gestiona el estilo del documento que ha sido perdido al guardarlo en texto plano.

Para tratar los documentos con PIO5 no hace falta conocer en profundidad html. En lugar de trabajar con el propio código, PIO5 cuenta con una barra de herramientas, similar a la de un procesador de texto, que facilita la inclusión de enlaces, la activación de direcciones electrónicas, la alineación y marcas del texto, etc. Así, se logra que la corrección o modificación del estilo en html sea una tarea cómoda y familiar para cualquier usuario. Teniendo en cuenta que fue diseñado sobre la base de una larga experiencia, esta herramienta va creando, a medida que se delimitan las secciones, un "árbol" a la izquierda del área de trabajo que sirve de guía para saber qué parte del texto se ha trabajado.

Como resultado, PI05 transforma los documentos y cada una de sus partes en un archivo único de extensión xml donde reúne los elementos necesarios para su procesamiento en Greenstone:

- Un archivo html, que contiene el documento completo con los metadatos de cada sección.

- Un archivo xml con los metadatos del documento general.

- Un archivo de imagen (la portada del documento).

Es decir, en el marco del proceso de descentralización del trabajo para la incorporación de libros, documentos y artículos en la red de bibliotecas virtuales, PIO5 permitió que todo el material pueda ser enviado en un solo archivo xml.

Finalmente, esta herramienta nos permite ampliar el servicio de textos completos de la red, brindando acceso a mayor cantidad de documentos cada mes porque cada vez son más los centros cooperantes que trabajan a distancia preparando sus propios documentos para Greenstone.

\section{Trabajo con metadatos}

Al detenernos a reflexionar sobre la aplicación práctica que Greenstone significa para esta biblioteca virtual cooperativa, resulta imprescindible pensar en el trabajo vinculado con los metadatos temáticos. La alternativa que brinda esta biblioteca cooperativa para el procesamiento y publicación de textos permite acceder a los últimos desarrollos en temas vinculados a las ciencias sociales que son generados por los centros miembros de Clacso.

Por tratarse de una biblioteca especializada en ciencias sociales, se ofrece el valor agregado de la clasificación humana, que permite al usuario realizar una búsqueda temática bien precisa. Por otra parte, para quienes analizan los textos, esta clasificación implica un trabajo creativo e intelectual de permanen- 
te especialización y perfeccionamiento.

Existen diferentes formas de localizar la información dentro de una biblioteca. La búsqueda en texto completo es el trabajo que hace automáticamente el programa; mientras que las consultas por título, autor, tema, es decir mediante los metadatos, requieren indización manual.

\section{"La aplicación práctica de Greenstone hace que pensar en el trabajo con metadatos temáticos sea imprescindible"}

Uno de los medios de acceso a la publicación es a través de una búsqueda por tema o temas, ya que a partir de las palabras asignadas a cada texto se agruparán los documentos por temáticas.

Las palabras clave o descriptores asignados para cada texto provienen de un proceso de reflexión interdisciplinario cuya premisa es la selección de hasta diez descriptores como máximo para cada documento. La tarea, que se realiza desde la Oficina coordinadora de la biblioteca cooperativa, junto con los centros cooperantes, intenta establecer conceptos comunes o generales que sean comprensibles para toda la región. Esta actividad se encuentra ligada a la utilización de un lenguaje específico y controlado, recurriendo en este caso al Macrothesauro de la OCDE (Macro) ${ }^{8}$. Éste es nuestro sustento de trabajo ya que es el único material de clasificación específico de ciencias sociales que incluye descriptores de diversas disciplinas del área.

Frente al constante desarrollo de las diferentes disciplinas de ciencias sociales, y debido a que la última actualización del Macrothe- sauro fue realizada en 1991, surgió la necesidad de enriquecer la terminología mediante la creación de un listado de descriptores libres ${ }^{9}$. Es utilizado como complemento al proceso de asignación de descriptores, es decir, se consulta cuando el Macro no posee descriptores puntuales o términos modernos para el tema tratado.

Cuando el texto que se analiza hace referencia a un lugar geográfico en particular se asigna la región, el país, la provincia y la ciudad (ejemplo: "América Latina, Bolivia, La Paz") porque, como se trata de una biblioteca virtual, el usuario puede acceder desde cualquier parte del mundo y no conocer las ubicaciones específicas de América Latina y Caribe.

Como muestra veremos los descriptores generales asignados para el texto Los tormentos de la materia. Aportes para una ecología política latinoamericana de Héctor Alimonda (compilador): "Ecología política, Gestión ambiental, Política ambiental, Recursos hídricos, Canal de Panamá, Medio ambiente, Desarrollo sustentable, América Latina, Caribe".

Asimismo podemos apreciar la diversidad temática en el interior de dos de sus capítulos y los descriptores temáticos analíticos propuestos para cada caso:

- "III. Teoría crítica y crítica política en la cuestión ambiental: problemas y perspectivas de Enoch Adames-Mayorga: Ecología política, Medio ambiente, Ciencia y tecnología, Naturaleza, Intelectuales, Teoría política, América Latina, Caribe.

- X. Con la soja al cuello: crónica de un país hambriento productor de divisas de Domínguez, Diego; Sabatino, Pablo: Ecología política, Medio ambiente, Política agraria, Economía, Recursos naturales, Nueva tecnología, Soja, Transgénicos, América Latina, Argentina".
Los usuarios de esta biblioteca virtual cooperativa utilizan el servicio de búsqueda de información a través de internet. Por tal motivo, la respuesta que obtengan en su búsqueda estará ligada en gran parte a la calidad de la clasificación que se logre en cada texto. Es por esto que la asignación de metadatos ${ }^{10}$ se realiza analíticamente, es decir, cada capítulo o parte del texto tiene sus propios metadatos.

La mirada interdisciplinaria para la asignación de descriptores enriquece la tarea y tiende a enfatizar las distintas aristas desde las que un fenómeno social puede ser analizado.

\section{Conclusión}

Trabajar con software libre fue una decisión sencilla en la Red de Bibliotecas Virtuales de Ciencias Sociales de América Latina y el Caribe porque Clacso es una red académica comprometida con el uso de plataformas libres y el acceso a los textos digitales de las investigaciones que se realizan en los centros miembros de la red es abierto.

"Clacso es una red académica comprometida con el uso de plataformas libres y el acceso abierto a los textos digitales"

Esta filosofía es compartida por las agencias que apoyan financieramente los programas de la red Clacso: principalmente $A S D I$ (gobierno sueco), NORAD (gobierno noruego), IDRC (gobierno de Canadá) e Inasp (International Network for the Availability of Scientific Publications).

Cuando esta biblioteca virtual cooperativa comenzó, los centros miembros entendían muy poco de 
estas nuevas formas de producir $y$ difundir textos en formato digital. Por eso Clacso organizó actividades de promoción y capacitación del personal de las áreas de publicaciones, biblioteca e informática para que colaboraran en los nuevos sistemas de trabajo. Diez años después, el eje se ha invertido y este servicio se encuentra desbordado para atender la demanda de los centros que quieren difundir rápidamente la versión digital de las publicaciones, siendo cada vez más habitual que ésta sea la única versión difundida. Esto requiere una detallada revisión de procedimientos y programas utilizados para acelerar y simplificar los procesos. En esta tarea estamos trabajando actualmente.

\section{Notas}

1. http://www.clacso.org.ar/biblioteca

2. http://www.greenstone.org

3. http://www. plone.org
4. Xml: eXtensible Markup Language.

5. http://creativecommons.org

6. http://www.vim.org

7. http://sala.clacso.org.ar/biblioteca/pi05/pi05

8. http://bibliotecavirtual.clacso.org.ar/ar/oecdmacroth

9. http://sala.clacso.org.ar/biblioteca/Members/ claves/desclib/desliba

10. Guía de asignación de descriptores temáticos. Clasificación Temática o Indización: Humana:

http://bibliotecavirtual.clacso.org.ar/ar/bibliointra/documentacion/analisis_documental.pdf

\section{Bibliografía}

Babini, Dominique; Fraga, Jorge (comp.). "Edición electrónica, bibliotecas virtuales y portales para las ciencias sociales en América Latina y El Caribe". Buenos Aires: Consejo Latinoamericano de Ciencias Sociales, 2006, pp. 271. ISBN 987-1183-53-4.

http://bibliotecavirtual.clacso.org.ar/ar/libros/ secret/babini/babini.html

Babini, Dominique. "Producción, difusión y uso de investigación colaborativa en red: el caso de la red académica del Consejo Latinoamericano de Ciencias Sociales". En: Albornoz, Mario; Alfaraz, Claudio (eds.). Redes de conocimiento: construcción, dinámica y gestión. Buenos Aires: Red Iberoamericana de Indicadores de Ciencia y Tecnología (Ricyt), 2006, pp. 249-258.
http://www2.ricyt.org/docs/libroRC2006/libroRC2006.pdf

Garrido-Picazo, Piedad; Tramullas, Jesús. "Un experimento de creación de biblioteca digital con Greenstone”. En: El profesional de la información, 2004, marzo-abril v. 13, n. 2, pp. 84-92.

http://eprints.rclis.org/archive/00008299/

Jose, Sanjo. "Adoption of open source digital library software packages: a survey". En: Kumar, Manoj K., eds. Proceedings Caliber 2007: 5th International convention on automation of libraries in education and research institutions. Febrero 2007, pp. 98-102.

http://eprints.rclis.org/archive/00008750/

Tramullas, Jesús. "Software libre para gestión de recursos de información digital". En: Encuentro internacional sobre conocimiento libre, II Conferencia internacional de software libre, 2006, pp. 346-356

http://eprints.rclis.org/archive/00006272/

Dominique Babini, Florencia Vergara-Rossi, Flavia Medici y Jessica González, Oficina Coordinadora de la Red de Bibliotecas Virtuales de Ciencias Sociales de América Latina y el Caribe, Buenos Aires.

biblioteca@clacso.edu.ar

\section{nature.com}

cs cloncla
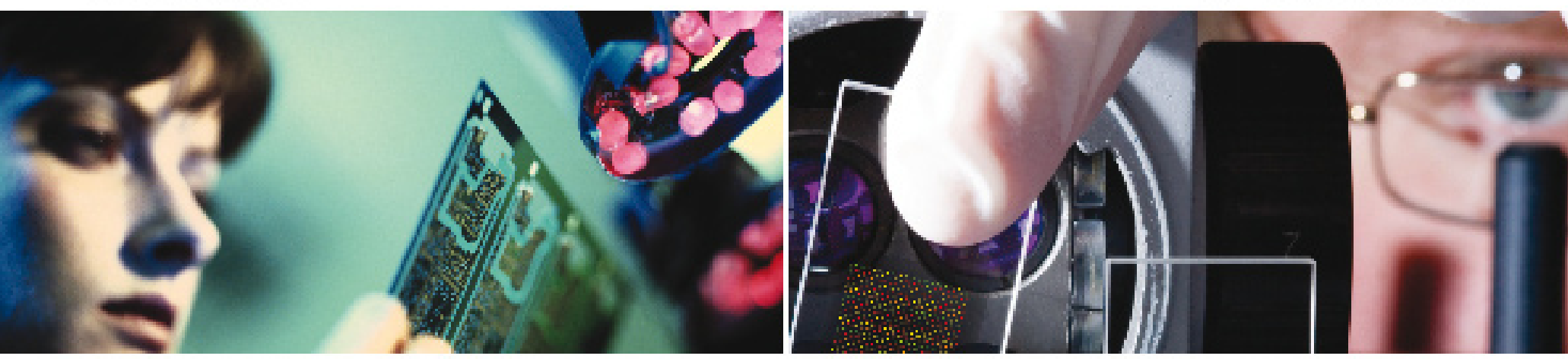

Todo esté y rijostro nature.com

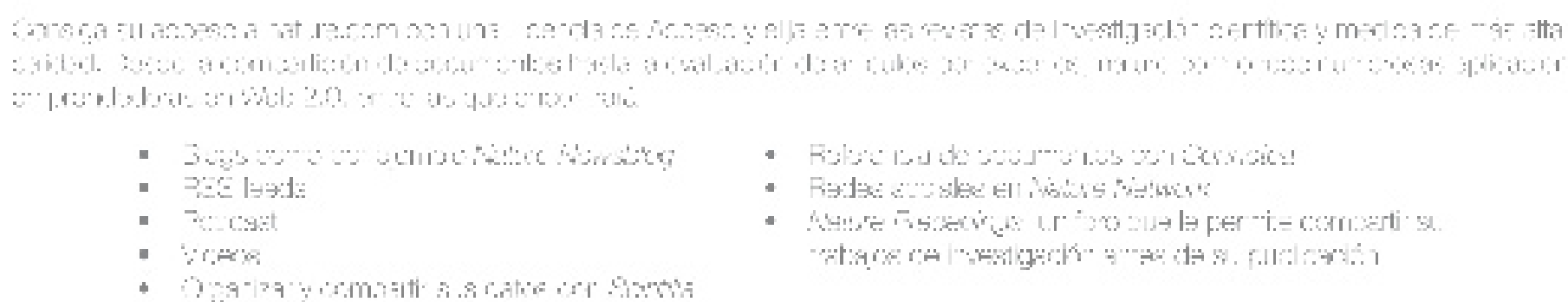

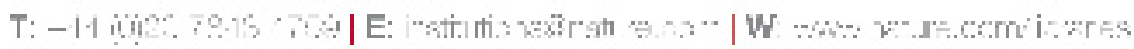

